\title{
Estudo comparativo da influência da razão de sopro nas propriedades de filmes tubulares de PEBD e PEAD
}

\section{Comparative study of the influence of blow-up ratio in the properties of HDPE and LDPE extruded blow films}

\author{
Roberto Luiz Rodriguez Ferreira ${ }^{1 *}$ e Ruth Marlene Campomanes Santana ${ }^{2}$ \\ ${ }^{1}$ Instituto Federal Sul-Rio-Grandense, Sapucaia do Sul, RS, Brasil \\ ${ }^{2}$ Departamento de Materiais, Universidade Federal do Rio Grande do Sul - UFRGS, \\ Porto Alegre, RS, Brasil \\ *rferreira@sapucaia.ifsul.edu.br
}

\begin{abstract}
Resumo
O objetivo deste trabalho é avaliar a influência do tipo de poliolefina e da razão de sopro (RS) nas propriedades físicas e térmicas de filmes produzidos em processo de extrusão tubular. No processamento foram utilizados um polietileno de baixa densidade (PEBD) e um polietileno de alta densidade (PEAD) transformados numa linha de extrusão de filme tubular com três RS de 1,5:1; 2,5:1 e 3,5:1. Os resultados das caracterizações físicas dos filmes mostraram maior variação de espessura no perímetro dos filmes tubulares de PEAD, quando comparados aos de PEBD. O aumento da RS causou uma diminuição de amplitude desta variação nos filmes tubulares de PEBD e diferenças nas propriedades ópticas. O coeficiente de atrito (COF) dos filmes foi menor para o PEAD e não teve variação significativa com o aumento da RS utilizada. Em relação às propriedades mecânicas, os filmes de PEAD apresentaram maior resistência à tração e menores valores de deformação no ponto de escoamento em ambas as direções. As RS maiores apresentaram similaridade nas tensões e nas deformações entre as direções da máquina (DM) e transversal (DT). A RS ideal, constatada para os filmes processados com PEBD, é 2,5:1 e com PEAD, é 3,5:1. Os filmes de PEAD apresentaram opacidade maior e menor brilho, quando comparados aos filmes de PEBD. Os resultados comprovaram valores conhecidos na literatura, entretanto introduziu-se uma inovação neste estudo: a utilização da técnica termográfica digital portátil, que permite visualizar, de maneira instantânea (on-line), as variações de temperatura no perímetro do balão, na zona de névoa (região de cristalização ou solidificação do filme).
\end{abstract}

Palavras-chave: PEBD, PEAD, PELBD, razão de sopro, linha de névoa, filme tubular, termográfica.

\begin{abstract}
The purpose of this study is to evaluate the influence of the polyolefin type and the blow-up ratio (BUR) on the thermal physical properties of films produced in a blown film extrusion process. It was used low density polyethylene (LDPE) and high density polyethylene (HDPE) processed in a blown film extrusion line at three BUR of: 1.5:1; 2.5:1 and 3.5:1. The results of physical characterization on these films demonstrated that the increase in the BUR shows a higher thickness variation in the perimeter of tubular films and, therefore, optical properties variation. The coefficient of friction (COF) of the films was smaller for HDPE and did not have significant variation with the increase in the BUR applied. Regarding mechanical properties, HDPE films showed higher tensile strength and lower strain values in the yield point, in both directions. The largest BUR showed higher similarity of the tensions and strains between the machine direction (MD) and transverse direction (TD). The ideal BUR observed for films processed with LDPE is $2.5: 1$, for HDPE this number is higher and close to 3.5:1. The HDPE films showed more opacities and less brightness when compared to LDPE films. The results prove well known values in the literature, however, there was an innovation introduced in this study which is the use of the portable digital thermo graphic technique that allows the instantaneous visualization (on-line) of the temperature variation in the bubble perimeter in the frost line (crystallization or solidification area of the film).
\end{abstract}

Keywords: LDPE, HDPE, LLDPE, blow-up ratio, frost line, blown film, thermographic. 


\section{Introdução}

Um dos principais processos de transformação de polietilenos é o de extrusão tubular de filmes. Empresas transformadoras necessitam produzir filmes tubulares com dimensionais diversos e com polímeros distintos, sendo os mais usados o PEBD, o polietileno linear de baixa densidade (PELBD) e o PEAD. Nem sempre os resultados são os esperados, seja por características do polímero utilizado, seja pela inadequação de equipamentos de que o transformador dispõe. Em geral são utilizadas as blendas destes materiais e não os componentes puros com o intuito de se obterem as melhores características exigidas do produto. Também há necessidade de suprir deficiências de equipamentos defasados em tecnologia ${ }^{[1]}$.

Na sua forma mais simples, a molécula de polietileno é constituída de átomos de carbono ligados covalentemente a um par de átomos de hidrogênio. As extremidades desta cadeia são terminadas por grupos metila ${ }^{[2-7]}$. Existem muitos tipos de polietileno, sendo que todos possuem essencialmente a mesma cadeia principal de átomos de carbono. As variações surgem, principalmente, a partir das ramificações que modificam as propriedades do material. Ocorrem muitos tipos de ramificações que vão desde simples grupos alquila a funcionalidades de ácido e éster. Em uma menor extensão, variações surgem a partir de defeitos na cadeia principal do polímero e que consistem de grupos vinila que estão freqüentemente associados às extremidades da cadeia. No estado sólido, ramificações e outros defeitos na estrutura da cadeia normal limitam o nível de cristalinidade da amostra ${ }^{[8]}$. As cadeias que têm poucos defeitos têm um grau mais elevado de cristalinidade do que aquelas que possuem muitos ${ }^{[2-7]}$

Como o empacotamento de regiões cristalinas é maior do que o das regiões não cristalinas, a densidade global de uma resina de polietileno está relacionada ao seu percentual de cristalinidade. Geralmente, quanto maior é a concentração de ramificações menor a densidade do sólido. As classes principais de polietileno, objetivo deste estudo, são ilustradas esquematicamente na Figura 1. O PEAD possui poucas e pequenas ramificações, conforme Figura $1 \mathrm{a}$, com densidades entre 0,94 e $0,97 \mathrm{~g} / \mathrm{cm}^{3}$ de acordo com Peacock ${ }^{[2]}$. O PEBD é um polímero ramificado formado por uma mistura de longas moléculas de diferentes tamanhos, ramificações de comprimentos variados e ramificações secundárias. Esse polímero possui variadas características que dependem do grau de ramificação ou da distribuição da massa molar, sendo que tais propriedades estão relacionadas às condições de reação da produção. A concentração significativa de ramificações dificulta o processo de cristalização, resultando em densidades relativamente baixas na faixa de 0,91 a $0,94 \mathrm{~g} / \mathrm{cm}^{3[2]}$.
Uma representação simplificada da estrutura do PEBD é mostrada na Figura 1b. As resinas de PELBD constituem-se de moléculas com cadeia principal de polietileno linear, que estão ligadas a grupos alquila curtos a intervalos aleatórios e cuja densidade encontra-se na faixa entre 0,90 e $0,94 \mathrm{~g} / \mathrm{cm}^{3[2]}$. Sua estrutura geral é representada na Figura 1c.

O tamanho de uma molécula de polietileno é normalmente descrito em termos de sua massa molecular média e de sua distribuição ${ }^{[2]}$. A massa molecular tem um efeito significativo sobre as propriedades físicas das resinas de polietileno, afetando a viscosidade, a resistência ao stress cracking - ESCR - quebra sob tensão ambiental - e ao impacto. No estado sólido, o material contém algumas regiões cristalinas, de curto alcance, permeadas por regiões amorfas, isto é, o polietileno encontra-se na forma semicristalina.

Os vários tipos de polietileno apresentam uma vasta gama de propriedades e atributos específicos que dependem das características moleculares e morfológicas da resina e das condições de processamento.

Os filmes de polietileno foram produzidos por processo de extrusão tubular que possui, como um dos principais parâmetros de controle, a RS utilizada no processamento. As aplicações desses filmes são diversas, destacando-se, entre outras: as de embalagens, sacarias, sacolas, filmes para construção civil, filmes e estufas agrícolas, silagem de grãos, biodigestores, geomembranas. Em todas elas, as propriedades mecânicas, térmicas e ópticas são muito importantes, porque se relacionam com a estrutura química do polietileno e com as condições de processamento utilizadas.

Nesse sentido, este estudo focaliza-se na análise das estruturas e propriedades finais de filmes processados por extrusão tubular com PEBD e PEAD em RS distintas.

\section{Experimental}

\subsection{Materiais}

Os polímeros utilizados foram o PEBD com índice de fluidez de $0,27 \mathrm{~g} / 10 \mathrm{~min}\left(190^{\circ} \mathrm{C} / 2,16 \mathrm{~kg}\right)$ e o PEAD com índice de fluidez de $0,58 \mathrm{~g} / 10 \mathrm{~min}\left(190^{\circ} \mathrm{C} / 5 \mathrm{~kg}\right)$, valores obtidos das fichas de produto, ambos fornecidos pela Braskem.

\subsection{Processamento}

O processamento dos filmes foi realizado numa linha de extrusão marca Carnevalli, modelo B700, Série B, ano 1985, programado para condições similares a fim de evidenciar as características das distintas formulações. Priorizaram-se os

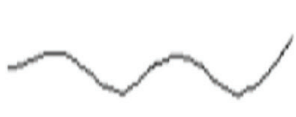

(a)

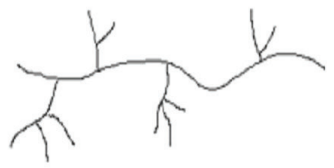

(b)

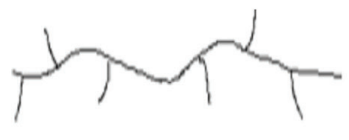

(c)

Figura 1. Esquema de diferentes classes de polietileno. (a) polietileno de alta densidade, (b) polietileno de baixa densidade, (c) polietileno linear de baixa densidade ${ }^{[1]}$. 
padrões de estabilidade e aparência dos filmes conjugados com o objetivo de obter os dimensionais projetados. Entretanto, durante o processamento, alteraram-se ajustes na altura da linha de névoa e na velocidade de tiragem necessárias para se adequarem às condições do equipamento e do processamento. As condições de processamento são apresentadas na Tabela 1 e as composições dos filmes, na Tabela 2.

\subsection{Caracterização}

A largura dos filmes foi medida próximo da bobinadeira no início, metade e final do processamento. A espessura deles foi obtida na passagem da torre para a bobinadeira, sempre no mesmo lado do filme (lado direito de extrusão), com um micrômetro marca Mitutoyo, modelo NO. 7301. As medições de largura e de espessura realizaram-se de acordo com as normas ASTM D6988-08 ${ }^{[9]}$ e ABNT NBR $15560-1^{[10]}$, respectivamente. $\mathrm{O}$ coeficiente de fricção (COF) dos filmes ${ }^{[11]}$ foi determinado no equipamento TMI (Testing Machines Inc.) conforme a norma ASTM D-1894 ${ }^{[12]}$. O ensaio mecânico de tração dos filmes foi realizado numa máquina de tração universal INSTRON, modelo 3367, de acordo com a norma ASTM D882-09, com o filme posicionado na DM e na DT, com célula de carga de $1 \mathrm{kN}$ e velocidade de ensaio de $500 \mathrm{~mm} / \mathrm{min}$.

A análise térmica realizou-se em um calorímetro diferencial exploratório (DSC), marca Perkin Elmer, modelo Pyris 6. As amostras dos filmes - aproximadamente $5 \mathrm{mg}$-, em atmosfera inerte $\left(\mathrm{N}_{2}\right)$ conforme norma ASTM D3418-99 ${ }^{[13]}$, foram submetidas à seguinte rotina:

- isoterma de 1 minuto a $20^{\circ} \mathrm{C}$;

- primeiro aquecimento de 20 a $200^{\circ} \mathrm{C}$ com taxa de $10^{\circ} \mathrm{C} /$ $\min$;

- isoterma de 1 minuto a $200^{\circ} \mathrm{C}$;

- resfriamento de 200 a $20^{\circ} \mathrm{C}$ com taxa de $10^{\circ} \mathrm{C} / \mathrm{min}$;

- isoterma de 1 minuto a $20^{\circ} \mathrm{C}$;

Tabela 1. Dados de processamento dos filmes tubulares.

\begin{tabular}{|c|c|c|c|}
\hline \multirow{5}{*}{$\begin{array}{l}\text { Temperaturas } \\
\left({ }^{\circ} \mathrm{C}\right)\end{array}$} & \multirow{3}{*}{ Extrusora } & Zona 1 & 200 \\
\hline & & Zona 2 & 200 \\
\hline & & Zona 3 & 200 \\
\hline & \multirow{2}{*}{\multicolumn{2}{|c|}{ Cabeçote/matriz }} & 200 \\
\hline & & & 200 \\
\hline \multicolumn{3}{|c|}{ Velocidade da rosca em todos os filmes (rpm) } & 70 \\
\hline \multirow{2}{*}{\multicolumn{2}{|c|}{ Velocidade inicial de tiragem $(\mathrm{m} / \mathrm{min})$}} & Filme 1 & 5,0 \\
\hline & & Filme 5 & 6,5 \\
\hline
\end{tabular}

Tabela 2. Composição e simbologia dos filmes tubulares processados.

\begin{tabular}{ccc}
\hline Formulação (PEBD/PEAD) & ${ }^{*}$ RS & Simbologia \\
\hline PEBD & $1,5: 1$ & $1 \mathrm{~A}$ \\
PEBD & $2,5: 1$ & $1 \mathrm{~B}$ \\
PEBD & $3,5: 1$ & $1 \mathrm{C}$ \\
PEAD & $1,5: 1$ & $5 \mathrm{~A}$ \\
PEAD & $2,5: 1$ & $5 \mathrm{~B}$ \\
PEAD & $3,5: 1$ & $5 \mathrm{C}$ \\
\hline
\end{tabular}

*Razão de sopro: (diâmetro do balão/diâmetro da matriz).
- e, finalmente, segundo aquecimento de 20 a $200^{\circ} \mathrm{C}$ com taxa de $10^{\circ} \mathrm{C} / \mathrm{min}$.

O monitoramento da temperatura do fundido foi efetuado na saída da matriz com um pirômetro de tecnologia a infravermelho Raytec Raynger ST. Através da termografia avaliou-se a distribuição de temperaturas no filme da saída da matriz até a linha de névoa - na direção da máquina e no perímetro do balão. Para a realização do ensaio, utilizou-se um termovisor de marca Fluke, modelo Ti10.

Para a detecção dos cristalitos, na superfície dos filmes, empregou-se o microscópio ótico Zeiss, modelo Axio Scope A1, de acordo com a norma ASTM E2015-04 ${ }^{[14,15]}$. Para os ensaios ópticos, espectrofotômetro BYK Spectroguide. A opacidade dos filmes foi detectada em valores percentuais $(\%)$ e o brilho, com feixe a $60^{\circ}$, cujos valores são adimensionais. As análises foram baseadas nas normas ASTM D1746-03, ASTM D 1003-00 e ASTM D 2457-97 ${ }^{[16-18]}$.

As amostras dos resultados, referenciadas em tabelas e gráficos, representam a média de 7 à 12 espécimes que foram ensaiadas em cada composição, de acordo com as respectivas normas ${ }^{[19]}$.

\section{Resultados e Discussões}

\subsection{Propriedades físicas}

$\mathrm{Na}$ Tabela 3, apresentam-se os parâmetros de processamento e os resultados das espessuras do filme nas diferentes composições. Constatou-se então que não foi atingida a especificação para a distribuição da espessura, definida assim: média de $60 \mu \mathrm{m}$ e desvio de $+/-5 \%$ conforme as exigências da norma ABNT NBR 15560-1 ${ }^{[10]}$. Isso também acontece com os equipamentos nas indústrias de transformação de filmes plásticos, produzindo uma situação real. Observa-se também que o valor da altura de linha de névoa do filme de PEBD oscilou de 280 a $320 \mathrm{~mm}$ e que a do PEAD permaneceu constante em $400 \mathrm{~mm}$. Em relação aos valores das espessuras dos filmes, o PEAD processado nas três RS e o PEBD na RS de 1,5:1 apresentaram maior variação que os filmes de PEBD nas RS de 2,5:1 e 3,5:1, o que pode ser constatado na Figura 2.

Para uma melhor visualização da forma dos filmes tubulares dos diferentes polímeros na saída da matriz, são mostradas as imagens desses filmes durante o processamento, na Figura 3, com suas correspondentes alturas de linhas de névoa assinaladas. Observa-se a influência do tipo de polímero e da RS na forma e largura dos filmes, entre a saída da matriz e o limite da altura da linha de névoa. A formação de pescoço foi mais pronunciada no PEAD. É possível observar-se também o aumento da opacidade do filme de PEAD, já que este é um polímero altamente cristalino, quando comparado ao PEBD, o que proporciona o maior empacotamento de suas cadeias ${ }^{[20]}$.

Os filmes de PEBD caracterizam-se por sua flexibilidade e resistência, deve-se isso à maior massa molar e à maior concentração de ramificações de diversos tamanhos, o que resulta no baixo grau de cristalinidade ${ }^{[21]}$. A tenacidade é o resultado de uma boa combinação de resistência e alongamento, particularmente, quando processado com alta 
Ferreira, R. L. R., \& Santana, R. M. C.

Tabela 3. Parâmetros e resultados de processamento dos filmes tubulares.

\begin{tabular}{|c|c|c|c|c|c|c|c|}
\hline \multicolumn{2}{|c|}{ Identificação do filme } & $\mathbf{1 A}$ & 1B & $1 \mathrm{C}$ & $5 \mathbf{A}$ & 5B & $5 \mathrm{C}$ \\
\hline \multicolumn{2}{|c|}{ Temperatura da massa $\left({ }^{\circ} \mathrm{C}\right)^{*}$} & 198 & 201 & 203 & 220 & 220 & 220 \\
\hline \multicolumn{2}{|c|}{ Largura do filme tubular (mm) } & 190 & 315 & 440 & 190 & 315 & 440 \\
\hline \multicolumn{2}{|c|}{ Altura da linha de névoa (mm) } & 280 & 320 & 320 & 400 & 400 & 400 \\
\hline \multicolumn{2}{|c|}{ Velocidade da rosca (rpm) } & 70 & 70 & 70 & 70 & 70 & 70 \\
\hline \multicolumn{2}{|c|}{ Velocidade de tiragem (m/min) } & 6,1 & 5,0 & 4,2 & 8,0 & 6,5 & 4,4 \\
\hline & 1 & 100 & 50 & 45 & 85 & 40 & 50 \\
\hline & 2 & 90 & 50 & 45 & 85 & 40 & 45 \\
\hline & 3 & 110 & 55 & 60 & 85 & 20 & 40 \\
\hline & 4 & 95 & 55 & 60 & 55 & 25 & 45 \\
\hline & 5 & 95 & 60 & 60 & 40 & 30 & 50 \\
\hline & 6 & 90 & 60 & 60 & 60 & 40 & 70 \\
\hline & 7 & 70 & 55 & 60 & 50 & 70 & 65 \\
\hline & 8 & 80 & 70 & 60 & 45 & 60 & 70 \\
\hline & 9 & 70 & 70 & 55 & 50 & 60 & 50 \\
\hline & 10 & 65 & 70 & 40 & 35 & 50 & 60 \\
\hline \multirow{8}{*}{ Espessura rilme $(\mu \mathrm{m})$} & 11 & 60 & 65 & 50 & 35 & 50 & 30 \\
\hline & 12 & 60 & 65 & 50 & 50 & 70 & 30 \\
\hline & 13 & 70 & 60 & 55 & 60 & 50 & 20 \\
\hline & 14 & 75 & 55 & 45 & 75 & 50 & 35 \\
\hline & 15 & 80 & 60 & 40 & 80 & 40 & 40 \\
\hline & 16 & 95 & 60 & 40 & 80 & 30 & 70 \\
\hline & Média real & 82 & 60 & 52 & 61 & 45 & 48 \\
\hline & Desvio padrão & 15 & 7 & 8 & 18 & 15 & 16 \\
\hline \multirow{3}{*}{$\begin{array}{l}\text { Limites de Especificação } \\
\qquad(\mu \mathrm{m})\end{array}$} & Superior $(+5 \%)$ & 63 & 63 & 63 & 63 & 63 & 63 \\
\hline & Inferior $(-5 \%)$ & 57 & 57 & 57 & 57 & 57 & 57 \\
\hline & Média & $=>>60$ & $=>>60$ & $=1>60$ & $=>>60$ & $=>>60$ & $=1>60$ \\
\hline
\end{tabular}

*Temperatura de massa medida com pirômetro Raytek Raynger ST na saída da matriz.

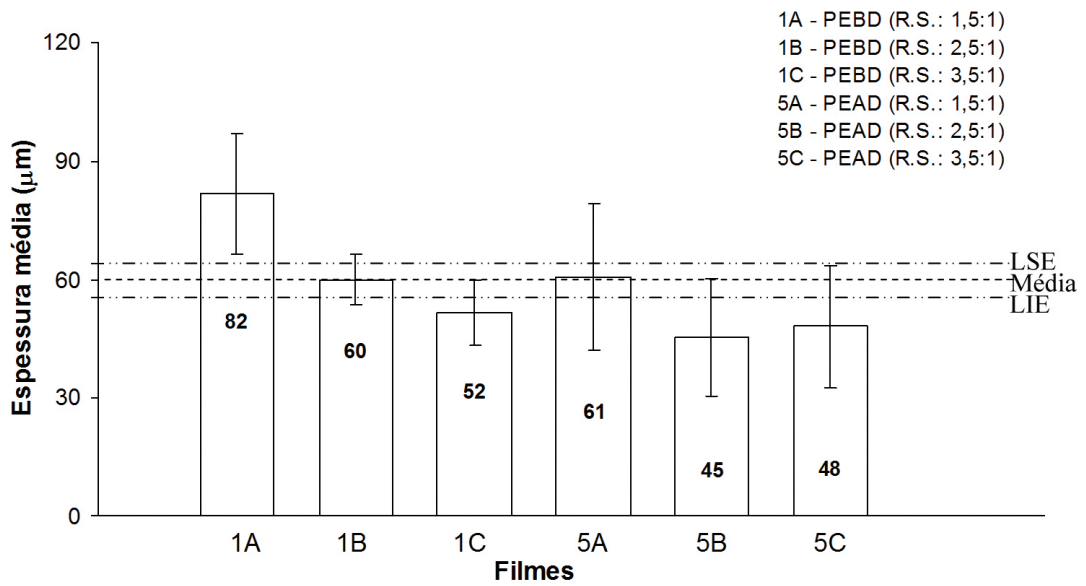

Figura 2. Espessura média e desvios dos filmes processados, em que LSE = limite superior de especificação, LIE = limite inferior de especificação, e Média = média de especificação $(60 \mu \mathrm{m})$.

orientação na DT. Uma das diferenças mais pronunciadas entre o processamento de filmes tubulares das poliolefinas avaliadas é que o PEAD apresenta uma altura de linha de névoa maior do que a do PEBD. O PEAD tende a ter uma menor resistência do fundido do que o PEBD causando maior instabilidade do balão ${ }^{[20]}$. Porém ocorre atraso no estiramento transversal do balão até que o material fundido esteja mais resfriado, resultando em uma linha de névoa elevada. Por outro lado, o PEAD tem a mais elevada resistência e rigidez em relação aos outros polietilenos, devido a seu melhor empacotamento entre as cadeias, com energias de ligação inter e intramolecular grandes, refletindo-se num maior grau de cristalinidade. Isso resulta num progresso contínuo da redução da espessura dos produtos de $\mathrm{PEAD}^{[21]}$.

\subsection{Análise da dispersão de espessuras dos filmes}

Tendo em vista que a espessura do filme influencia diretamente os resultados das análises, selecionaram-se as amostras com espessura próxima dos $60 \mu \mathrm{m}$. Na 

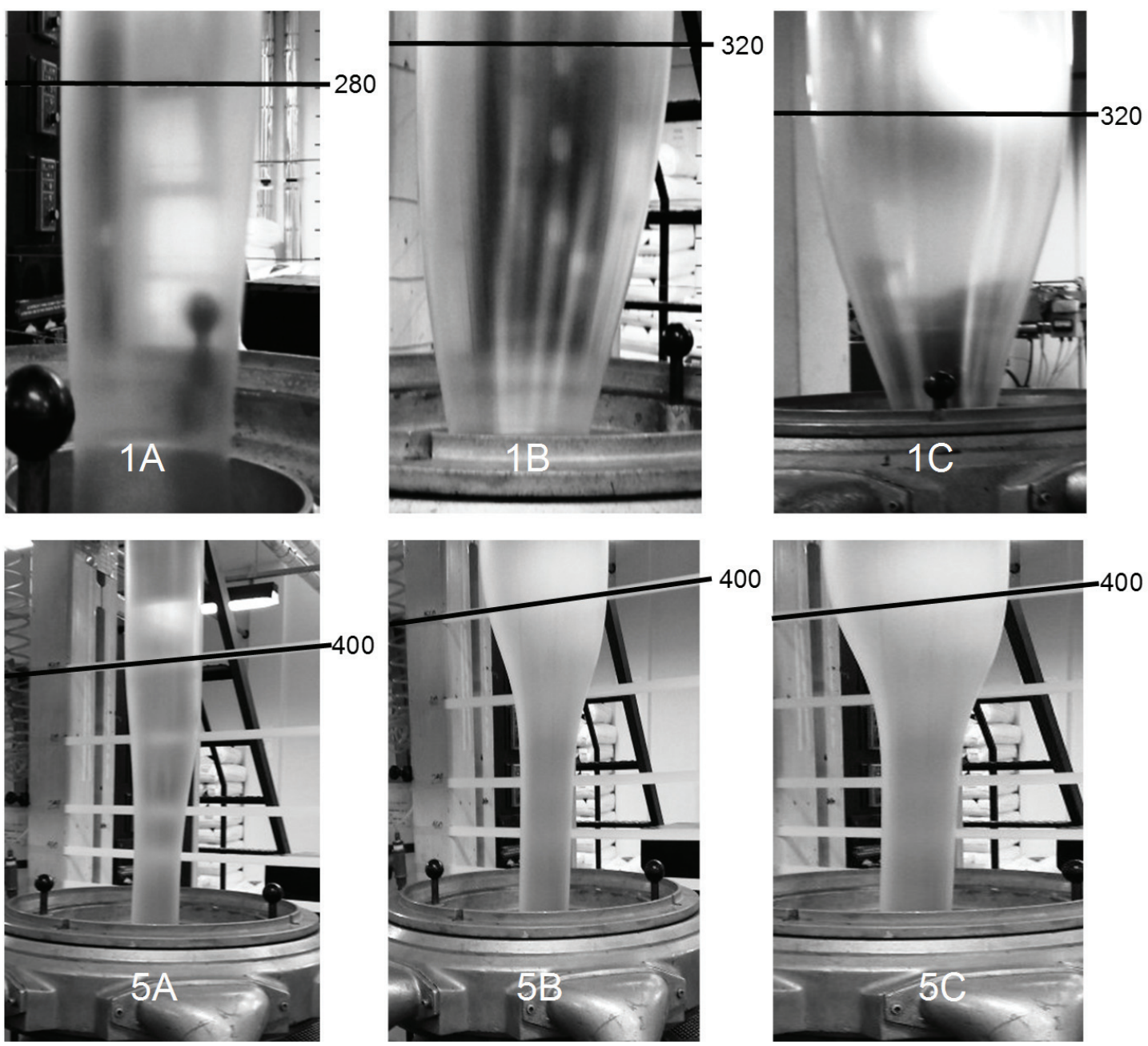

Figura 3. Imagens dos filmes tubulares com registro das alturas das linhas de névoa: 1A:280mm, 1B e 1C: 320mm, 5A, 5B e 5C: 400mm.

Tabela 3, observa-se uma grande variação de espessuras no perímetro dos filmes processados. Para visualizar melhor as diferenças, na Figura 2 mostra-se um comparativo das espessuras médias e os desvios dos filmes processados. Constatou-se que os filmes de PEBD (1B e 1C) apresentam uma distribuição mais homogênea da espessura (menor desvio em relação à média real) quando comparada a dos outros filmes de PEBD (1A) e de PEAD (5A, 5B e $5 \mathrm{C})$, mostrando-se ser a melhor correspondente ao filme processado com a RS de 2,5:1 (1B), embora esteja fora da especificação da norma ABNT NBR 15560-1 ${ }^{[10]}$.

Verificou-se também que a cada ajuste de RS ocorreram variações de espessura no perímetro do filme tubular. Isso pode estar relacionado a que em filmes tubulares o projeto da matriz e do anel de ar de resfriamento, as características reológicas do polímero utilizado e as condições de processamento, influenciam sobremaneira o comportamento do fluxo do material fundido pela matriz e a posterior fixação de geometria do filme. É muito difícil obter-se um perfil de distribuição de espessura perfeitamente homogêneo e linear. Normalmente se constata o aspecto irregular na região da linha de névoa, sendo comum a denominação "dentes de vampiro", em chão de fábrica $^{[22-25]}$.

\subsection{Largura dos filmes}

Os resultados das medições da largura tubular dos filmes mostrados na Tabela 3 foram 190mm, 315mm e 440mm, correspondentes as RS de 1,5:1; 2,5:1 e 3,5:1, respectivamente, para ambos os polímeros. Os filmes não apresentaram variação significativa na largura já que os processamentos ocorreram unicamente para obtenção de amostras dos filmes, ou seja, é pouco tempo para que qualquer alteração possa ser percebida. Em produções industriais/comerciais, é normal ocorrerem flutuações pequenas na largura dos filmes devido a mudanças nas condições ambientais e nas variáveis de processamento com o decorrer do tempo. Estas tolerâncias estão previstas na norma ABNT NBR 15560-1 ${ }^{[10]}$.

\subsection{Coeficiente de fricção - COF}

Um dos ensaios indiretos para avaliar se houve influência da composição das amostras na rugosidade das superfícies interna e externa dos filmes tubulares é 
o coeficiente de atrito, COF. Os resultados de COF da superfície dos filmes em contato interno/interno e externo/ externo são apresentados na Figura 4. Observa-se que as amostras de PEBD (1A, 1B e 1C) apresentam resultados de $\mathrm{COF}$ interno/interno e externo/externo, superiores às amostras de PEAD (5A, 5B e 5C).

Com relação à RS, não foi possível distinguir alguma diferença significativa da influência deste parâmetro de processamento no valor do COF dos filmes. Observa-se também que o COF dos filmes de PEBD é geralmente maior nas superfícies internas do que nas externas, embora sejam estatisticamente similares. A diferença de valores dos coeficientes externo e interno dos filmes pode ser explicada, pois mesmo sendo o filme de paredes pouco espessas, o que facilita a transmissão de calor, ocorre um acréscimo da temperatura do ar interno do balão, que está aprisionado - sem renovação, causando um processo diferenciado de cristalização da superfície interna em relação ao da superfície externa.

De acordo com Willmouth ${ }^{[2]}$, dois motivos principais esclarecem a aspereza de superfície: irregularidades induzidas pelo fluxo e estruturas cristalinas projetadas. $\mathrm{O}$ fluxo é um efeito reológico conhecido, que pode finalmente ser correlacionado à estrutura molecular. Avalia-se que a aspereza da cristalização ocorra devido à formação de agregados cristalinos na superfície dos filmes ou próximos $\operatorname{dela}^{[27]}$.

White e Cakmak ${ }^{[27]}$ estudaram uma grande faixa de materiais semicristalinos (PEBD, PEAD, PELBD, PP e PBT) e amorfos (PS e PMMA), constatando que a cristalização é a razão principal para a aspereza da superfície, enquanto o efeito reológico desempenha um papel secundário, mas importante. Para os filmes soprados de PEBD convencional, a elasticidade da massa fundida é a principal causa da aspereza, ao mesmo tempo uma maior aspereza no PEAD é atribuída ao seu elevado grau de cristalização ${ }^{[28]}$.

\subsection{Propriedades mecânicas - ensaio de tração}

Na Figura 5, são apresentadas, para as amostras de PEBD (filmes $1 \mathrm{~A}$ - RS: 1,5:1; 1B - RS: 2,5:1 e 1C-RS: 3,5:1), as curvas de tensão versus a deformação relacionadas à razão de sopro, onde é possível observar que, para a RS: 1,5:1, a tensão na ruptura é maior na DM do que na da DT. Os filmes processados com RS: 1,5:1 e 2,5:1 apresentam maior resistência à tração na ruptura, na DM, do que aqueles com RS: 3,5:1. Para as RS de 2,5:1 e de 3,5:1, do filme de PEBD, as tensões e deformações na ruptura na $\mathrm{DM}$ e na DT mostram-se similares. De acordo com a literatura ${ }^{[20,29-42]}$, a RS ideal para o PEBD ocorre entre 2,5:1 e 3,5:1, proporcionando resultados de propriedades mecânicas mais homogêneas tanto na DM como na DT. Portanto, a RS de 2,5:1 e menores induzem a uma distribuição molecular orientada na DM com resultado de tensões maiores que as da DT.

Na Figura 6, são apresentadas as curvas de tensão versus deformação relacionadas a RS para as amostras de PEAD. Observa-se, no perfil de todas as curvas dos filmes na DM e na DT, o ponto de escoamento bem pronunciado. Os valores de tensões na ruptura e de tensões no escoamento resultam maiores do que os das amostras do PEBD, visto que o PEAD é mais cristalino que o PEBD. O PEAD apresenta microestrutura linear com poucas ramificações e segmentos cristalizáveis longos que proporcionam estruturas cristalinas melhor organizadas.

Os resultados do ensaio de tração das amostras do PEBD e PEAD, apresentados resumidamente na Tabela 4, permitem mostrar outras considerações. O módulo de elasticidade do PEBD apresenta valores similares que variam entre 278 e $283 \mathrm{MPa}$, o que demonstra não ocorrer influência significativa da RS, pois está mais atrelado às características da matéria-prima. No PEAD os módulos de elasticidade variam entre 784 e $1190 \mathrm{MPa}$, sendo tais valores superiores aos do PEBD, resultado relatado na literatura ${ }^{[32-39]}$. Também se verifica que na RS:1,5:1 não houve diferença significativa no valor dos módulos de

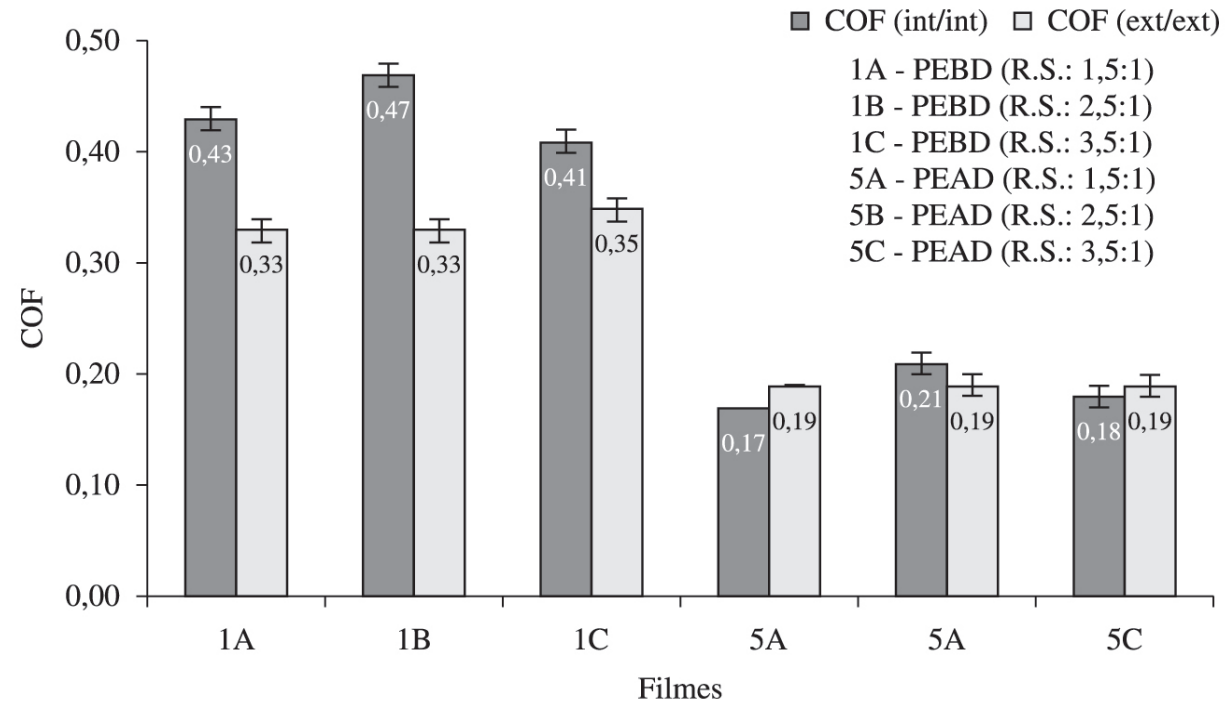

Figura 4. Resultados de coeficiente de fricção dinâmico interno/interno e externo/externo. 


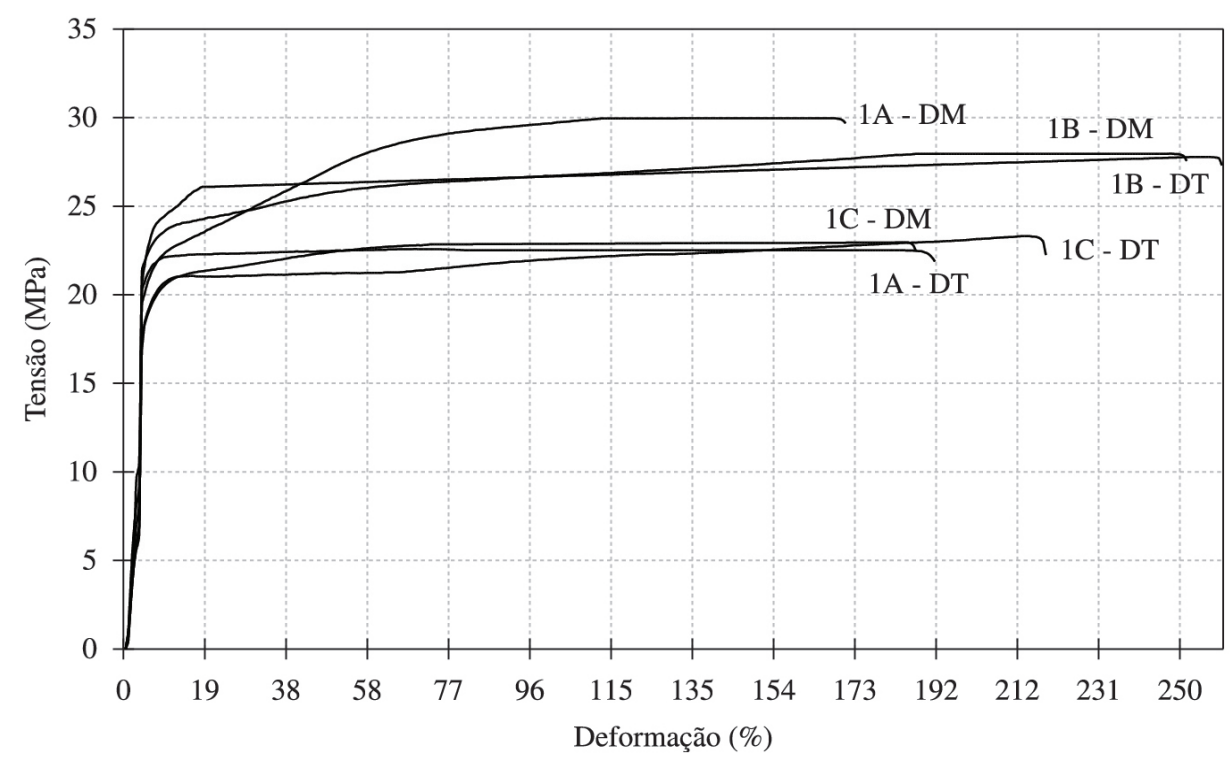

Figura 5. Curvas de tração versus deformação dos filmes de PEBD: $1 \mathrm{~A}$ - RS: 1,5:1; $1 \mathrm{~B}$ - RS: 2,5:1 e 1C - RS: 3,5:1. DM: direção de máquina, DT: direção transversal.

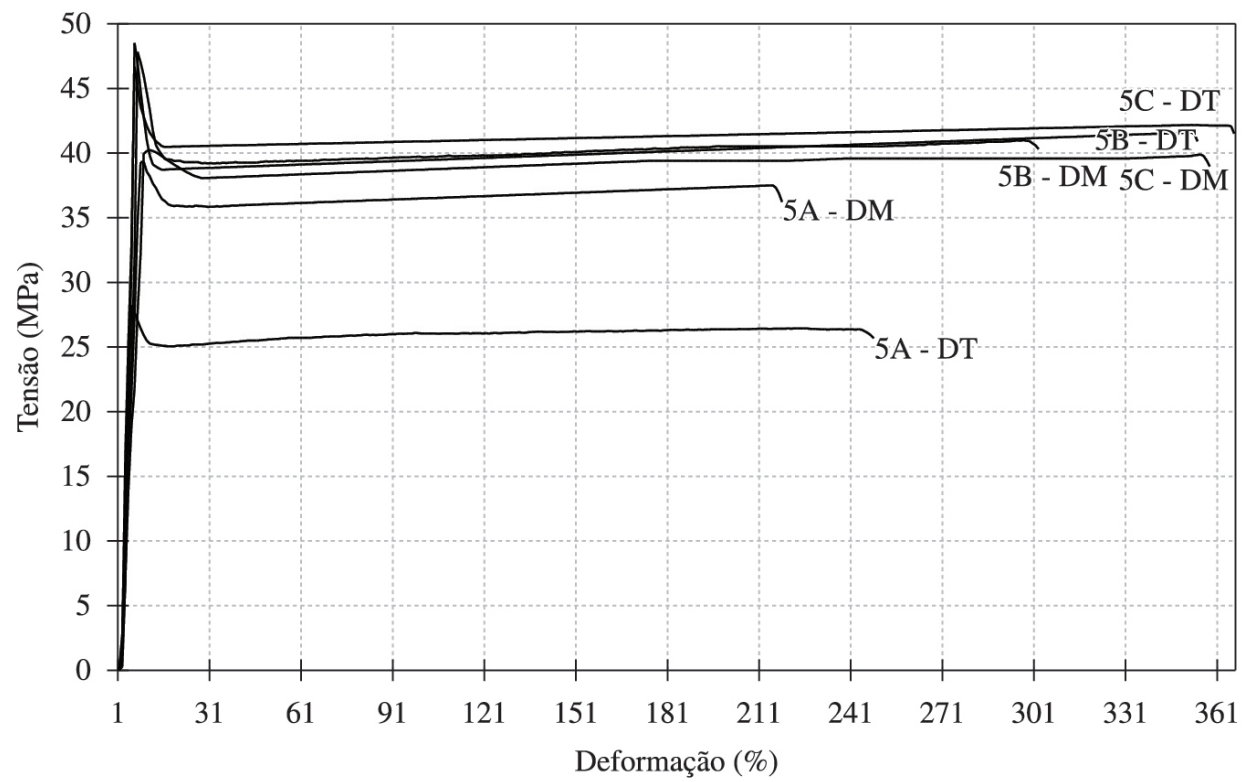

Figura 6. Curvas de tração versus deformação dos filmes de PEAD: 5A - RS: 1,5:1; 5B - RS: 2,5:1 e 5C - RS.: 3,5:1. DM: direção de máquina, DT: direção transversal.

Tabela 4. Dados de resistência à tração na ruptura, alongamento de ruptura e módulo dos filmes processados com PEBD e PEAD (A - RS: 1,5:1, B - R.S: 2,5:1 e C-R.S: 3,5:1).

\begin{tabular}{|c|c|c|c|c|c|c|c|c|c|c|c|c|}
\hline \multirow[t]{2}{*}{ Amostras } & \multicolumn{6}{|c|}{ PEBD } & \multicolumn{6}{|c|}{ PEAD } \\
\hline & \multicolumn{2}{|c|}{$\mathbf{1 A}$} & \multicolumn{2}{|c|}{ 1B } & \multicolumn{2}{|c|}{$1 \mathrm{C}$} & \multicolumn{2}{|c|}{$5 \mathbf{A}$} & \multicolumn{2}{|c|}{ 5B } & \multicolumn{2}{|c|}{$5 \mathrm{C}$} \\
\hline Razão de sopro & \multicolumn{2}{|c|}{$1,5: 1$} & \multicolumn{2}{|c|}{$2,5: 1$} & \multicolumn{2}{|c|}{$3,5: 1$} & \multicolumn{2}{|c|}{$1,5: 1$} & \multicolumn{2}{|c|}{$2,5: 1$} & \multicolumn{2}{|c|}{$3,5: 1$} \\
\hline Direção da amostra & DM & DT & DM & DT & $\mathrm{DM}$ & DT & $\mathrm{DM}$ & DT & DM & DT & DM & DT \\
\hline Tensão na ruptura(MPa) & 30 & 23 & 28 & 28 & 23 & 23 & 37 & 26 & 41 & 42 & 39 & 42 \\
\hline Deformação na ruptura (\%) & 168 & 184 & 247 & 254 & 185 & 213 & 137 & 157 & 191 & 225 & 228 & 233 \\
\hline Tensão no escoamento (MPa) & 20 & 20 & 21 & 21 & 24 & 26 & 39 & 28 & 48 & 49 & 40 & 47 \\
\hline Deformação no escoamento (\%) & 12 & 12 & 15 & 18 & 16 & 16 & 6 & 2 & 4 & 3 & 6 & 3 \\
\hline Módulo de elasticidade (MPa) ${ }^{*}$ & 282 & 280 & 283 & 282 & 278 & 279 & 787 & 784 & 944 & 1130 & 992 & 1190 \\
\hline
\end{tabular}

*velocidade de $500 \mathrm{~mm} / \mathrm{min}$. 
elasticidade da DM e da DT. Mas, para as RS maiores, há um aumento considerável do módulo, sendo mais significativo na DT.

No caso do PEBD, é possível notar-se que há um maior equilíbrio das tensões e deformações na ruptura em ambas as direções, DM e DT, para os filmes processados com a RS de 2,5:1. A RS ideal para filmes processados com PEBD deve estar entre 2,5:1 e 3,5:1, confirmando valores da literatura $^{[20,29-42]}$. Já para a RS de 1,5:1, os valores das tensões e deformações na ruptura foram menores (exceto o valor de tensão na ruptura para a DM de $30 \mathrm{MPa}$, o maior de todas as amostras) entre as direções DM e DT. Este resultado indica uma orientação molecular preferencial do filme processado na DM, em prejuízo da orientação molecular na DT, fato esperado pela baixa RS utilizada.

Já, no PEAD, os valores são os mais elevados e homogêneos para a RS: 3,5:1 (5C) demonstrando que uma RS mais elevada poderá conduzir a melhores resultados. Portanto a RS ideal para o PEAD deve ser próxima a 3,5:1. Da mesma forma que o PEBD, as amostras de PEAD também demonstraram valores maiores que os mínimos exigidos pela norma ABNT NBR $15560-1^{[10]}$ na ruptura, de $17 \mathrm{MPa}$ para a tensão e de $150 \%$ para a deformação.

O estudo realizado por Pucci e Shroff ${ }^{[28]}$, concluiu que a resistência máxima à ruptura é maior na $\mathrm{DM}$ do que na DT, cujo resultado foi confirmado por Patel et al. ${ }^{[29]}$ Neste estudo $^{[28]}$, também define que as tensões na DM e na DT são afetadas pela orientação das moléculas da fase amorfa.

\subsection{Termografia}

O termovisor Fluke Ti10 é originalmente projetado para a solução de problemas diários na manutenção de circuitos e painéis elétricos, sistemas de distribuição de energia elétrica, sistemas de ventilação, sistemas de climatização de ambientes, sistemas de refrigeração e equipamentos a estes associados. Ele auxilia os técnicos na análise e reparos destes sistemas. A sua utilização no acompanhamento do processo, desde a saída da massa fundida da matriz até a linha de névoa, é inovadora e representa um avanço na análise de processos de extrusão. O termovisor proporciona versatilidade em acompanhar várias linhas de extrusão com um só equipamento portátil, e agilidade na correção dos ajustes de folga da matriz e da vazão do anel de ar de resfriamento. A sua utilização representa um custo reduzido quando comparado aos sistemas usuais de medição de espessura em linha, que são incorporados a um único equipamento de extrusão.

O termovisor permite a definição exata da altura da linha de névoa que, na maioria das vezes, dificilmente enxerga-se a olho nu, facilitando a visualização das diferenças de temperatura captadas no perímetro do balão e correlacionando-as às diferenças de distribuição de espessura. Ou seja, pontos de maior temperatura são os de maior espessura e pontos de menor temperatura são os de menor espessura. O termovisor proporciona a percepção das distintas temperaturas da massa fundida de acordo com a formulação utilizada, leitura e coleta de dados de forma instantânea e a possibilidade de tratar os dados posteriormente, com um software específico dedicado ao instrumento. No caso deste estudo verifica-se o aumento da temperatura da massa fundida do PEAD, isto se deve ao comportamento reológico dos materiais envolvidos, havendo um maior cisalhamento com o PEAD e o conseqüente aumento da temperatura da massa fundida

As imagens obtidas com o termógrafo, Figura 7, e os dados, Tabela 5, permitem observar-se o perfil de temperatura do polímero a partir da sua saída da matriz até a região onde ocorre a sua solidificação (altura da linha de névoa) pela variação de tonalidade e intensidade das cores. Também confirma-se a distribuição distinta entre os processamentos e a variação da espessura em torno do perímetro do filme tubular (aspecto de "dentes de vampiro") pela configuração de tonalidades de cores. $\mathrm{Na}$ definição original do instrumento as diferenças ficam melhor definidas e observáveis, em cores.

\subsection{Microscopia Óptica - MO}

Na Figura 8, são apresentadas as imagens das superfícies das amostras obtidas por microscopia óptica. Observa-se o aumento da concentração de cristalitos do PEAD em comparação ao PEBD, tendo em vista que as suas microestruturas influenciam no processo de cristalização, devido ao PEAD apresentar cadeia linear mais organizada e seguimentos cristalizáveis longos, gerando estruturas cristalinas melhor organizadas/perfeitas, que se fundem/ cristalizam em temperaturas maiores. Com base nisto, observa-se a altura da linha de névoa maior para o PEAD, o que lhe permite mais tempo para a formação dos cristalitos e que eles se formem em maior quantidade, de acordo com a literatura ${ }^{[28]}$.

Observa-se a diminuição do tamanho dos cristalitos em filmes com maior RS (1C e 5C - 3,5:1), provavelmente, devido ao processo de bi-orientação, já que isto pode restringir o crescimento dos cristalitos, sendo mais pronunciado para o $\mathrm{PEBD}$, o que pode estar relacionado com a menor altura da linha de névoa. Constata-se também um maior número de cristalitos do PEAD com o aumento da RS, isso permite mais tempo para a formação dos cristalitos e que eles se formem em maior quantidade, como comprovado pela literatura ${ }^{[27]}$.

\subsection{Propriedades ópticas: opacidade e brilho}

Estas propriedades ópticas - opacidade e brilho - podem nos indicar características intrínsecas do material relacionadas à cristalinidade, ou à topografia da superfície da amostra. O mecanismo de resposta de

Tabela 5. Intervalos de temperaturas em relação à tonalidade de cores das imagens termográficas.

\begin{tabular}{cccc}
\hline \multirow{2}{*}{ Filme } & \multicolumn{3}{c}{ Faixa de temperaturas $\left({ }^{\circ} \mathbf{C}\right)$} \\
\cline { 2 - 4 } & $\begin{array}{c}\text { Cinza } \\
\text { translucido }\end{array}$ & Cinza leitoso & Cinza escuro \\
\hline 1A & $21-77$ & $77-112$ & $112-158$ \\
1B & $20-80$ & $80-112$ & $112-174$ \\
1C & $21-90$ & $90-117$ & $117-185$ \\
5A & $20-90$ & $90-140$ & $140-212$ \\
5B & $20-90$ & $90-150$ & $150-212$ \\
5C & $20-90$ & $90-160$ & $160-221$ \\
\hline
\end{tabular}



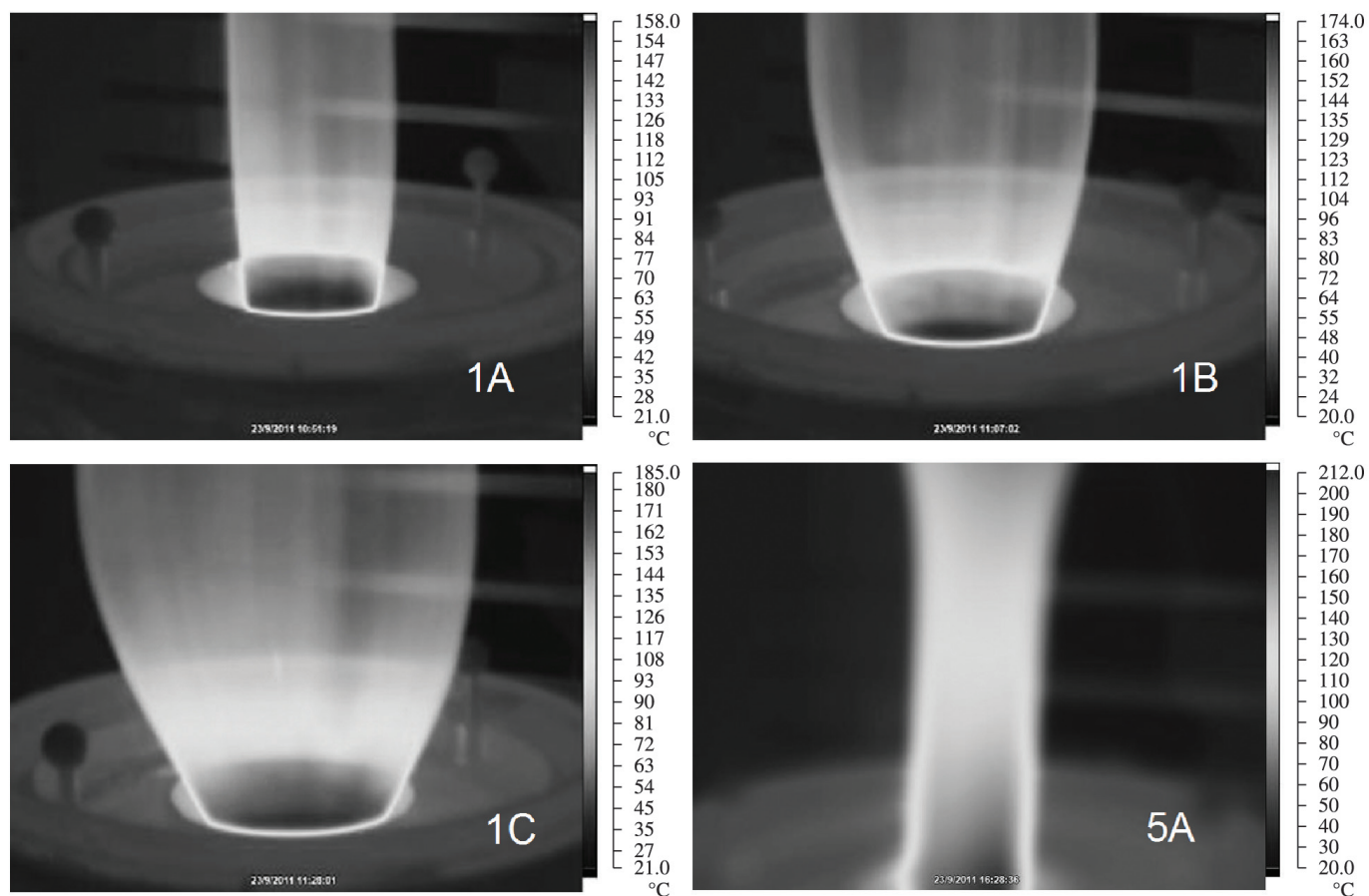

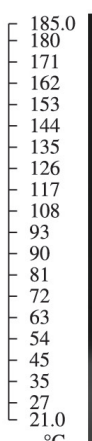
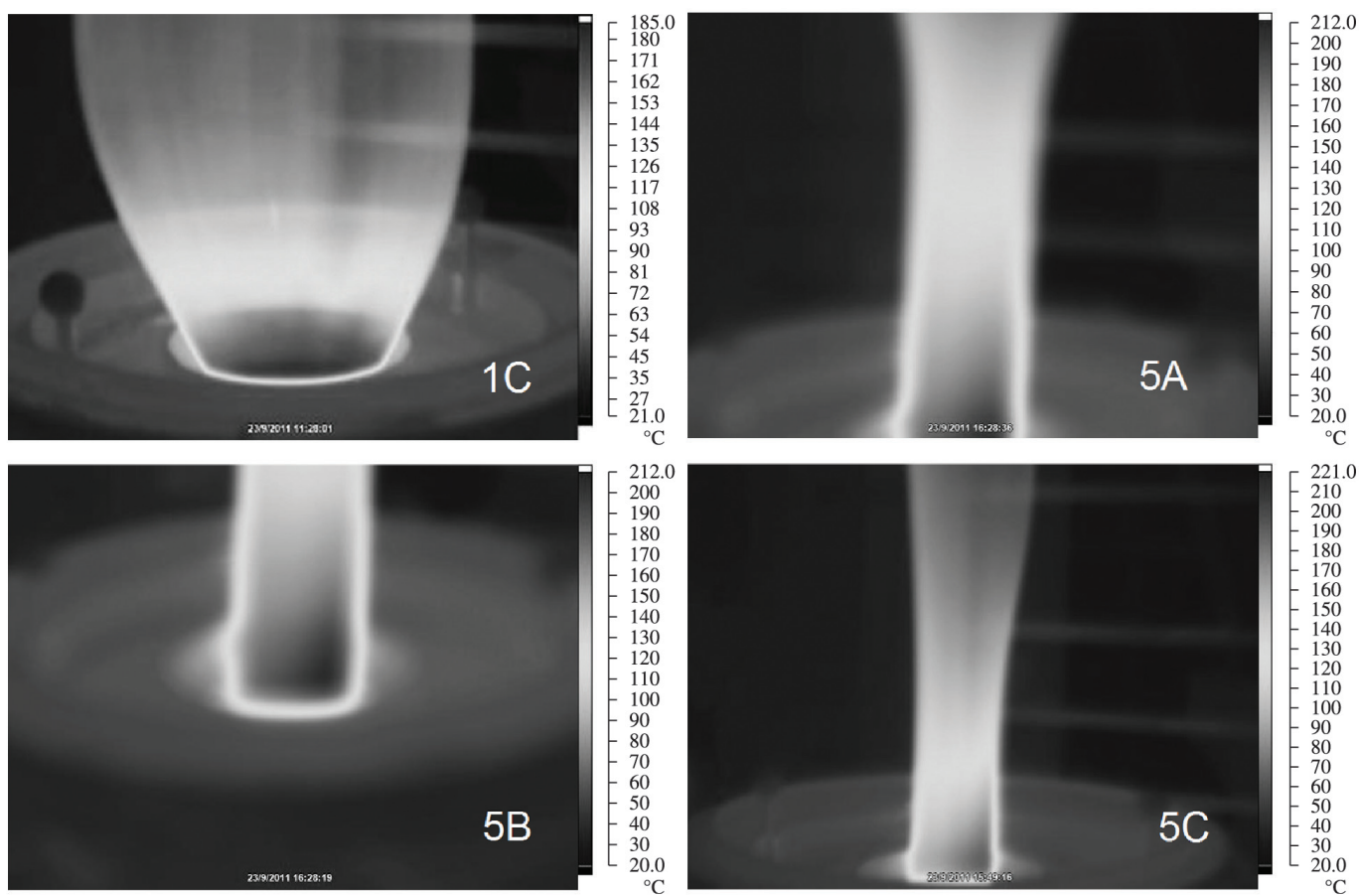

Figura 7. Imagens dos filmes tubulares com registro das temperaturas após a saída da matriz.

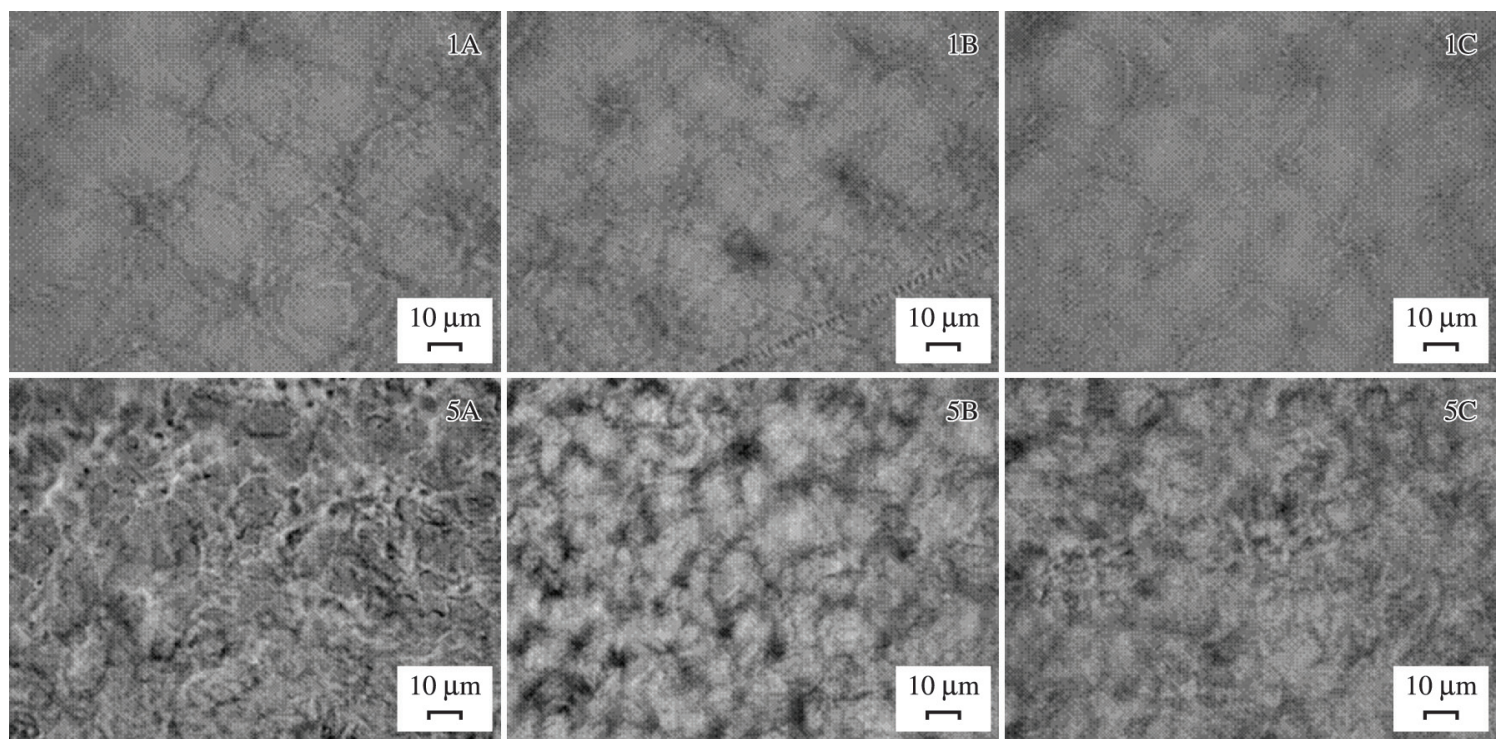

Figura 8. Imagens das amostras dos 6 filmes obtidas por microscopia óptica, com diminuição do tamanho dos cristalitos $(1 \mathrm{~A}>1 \mathrm{~B}>1 \mathrm{C}$ e $5 \mathrm{~A}>5 \mathrm{~B}>5 \mathrm{C})$ com o aumento da RS, sendo os do PEBD $<$ PEAD. 
tais propriedades pode ser fundamentado pelo fenômeno físico de absorção e transmissão da luz.

Na Figura 9, são mostradas as opacidades médias das amostras nas quais se observa que os filmes de PEAD apresentam opacidades maiores do que os de PEBD, característica específica de polímeros de alto grau de cristalinidade. Também é possível visualizar-se que no PEBD a queda da opacidade do material pode estar relacionada ao menor grau de cristalinidade deste, devido ao número de ramificações de maior tamanho que distancia as cadeias macromoleculares impedindo o empacotamento delas ${ }^{[2]}$. Em geral, os materiais com grau de cristalinidade maior apresentam opacidade maior, porque a transmissão direta da luz, através das amostras, é dificultada pela formação dos cristais ${ }^{[6]}$. O comportamento anômalo da amostra de 100\% PEAD - 5B pode ser atribuído a erro experimental ou imperfeições na superfície do filme ${ }^{[2]}$.

Na Figura 10, são apresentados os resultados do ensaio de brilho em que os filmes de PEBD apresentam maior valor que os de PEAD, reafirmando o resultado encontrado para opacidade, isto é, o aumento da cristalinidade do PEAD. Brilho e opacidade são propriedades que variam de forma inversamente

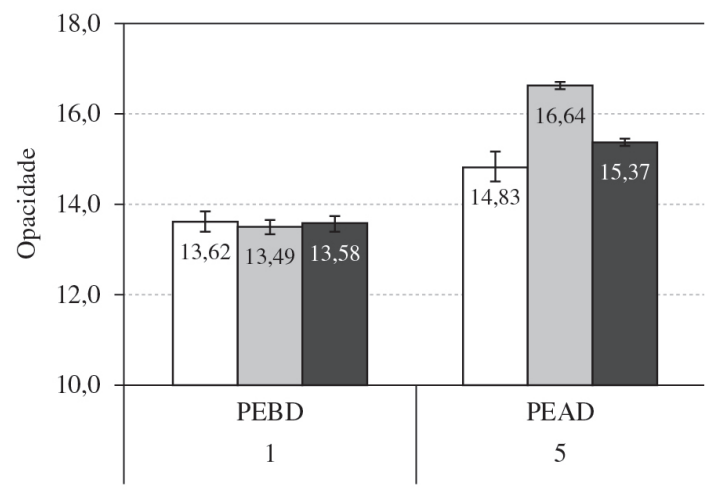

$\square$ A (RS: 1,5:1) $\square$ B (RS: 2,5:1) $\square$ C (RS: 3,5:1)

Figura 9. Opacidade dos filmes processados.

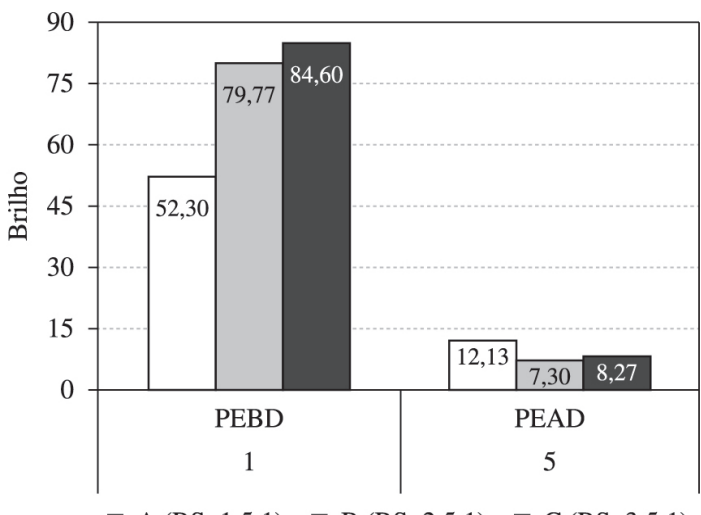

$\square$ A (RS: 1,5:1) $\square$ B (RS: 2,5:1) $\square$ C (RS: 3,5:1)

Figura 10. Brilho dos filmes processados. proporcional, o que é confirmado por Peacock ${ }^{[2]}$. Ainda, de acordo com Pucci et al., ${ }^{[28]}$ o tamanho dos cristalitos influencia o brilho e a opacidade do material. Conforme as Figuras 9 e 10, o filme de PEAD, apresenta cristalitos maiores, portanto opacidade maior e brilho menor.

\section{Conclusões}

Os resultados deste trabalho levaram a concluir que:

- as RS maiores possibilitaram maior similaridade das tensões e das deformações no escoamento entre as direções DM e DT;

- a RS ideal constatada para os filmes processados estabeleceu-se entre 2,5:1 e 3,5:1 para o PEBD e 3,5:1 para o PEAD;

- os resultados de microscopia óptica mostraram a diminuição do tamanho dos cristalitos nos filmes de PEBD e PEAD (1C e 5C) com maior RS (3,5:1), e um aumento da concentração no número de cristalitos do PEAD em relação ao PEBD;

- os filmes de PEBD apresentaram maior valor de brilho que os de PEAD;

- os resultados obtidos foram promissores, pois introduziram uma nova tecnologia para o acompanhamento do controle de processos de extrusão (on-line) com a utilização de um termógrafo portátil.

\section{Referências}

1. Liu, C., Wang, J., \& He, J. (2002). Rheological and Thermal Properties of m-LLDPE blends with m-HDPE and LDPE. Polymer, 43(13), 3811-3818. http://dx.doi.org/10.1016/S00323861(02)00201-X.

2. Peacock, A. J. (2000). Handbook of polyethylene: structures: properties and applications. New York: Marcel Dekker Inc.

3. Benham, E., \& MacDaniel, M. (2010). Ethylene polymers HDPE. Encyclopedia of Polymer Science and Technology, 2, 382-412.

4. Maraschin, N. (2010). Ethylene polymers - LDPE. Encyclopedia of Polymer Science and Technology, 2, 412-441.

5. Simpson, D. M., \& Vaughan, G. A. (2010). Ethylene polymers - LLDPE. Encyclopedia of Polymer Science and Technology, 2, 441-482.

6. Brydson, J. A. (1999). Plastics materials (7th ed.). Oxford: Butterworth-Heinemann.

7. Mikitaev, A. K., Ligidov, M. K., \& Zaikov Gennady, E. (2006). Polymers, polymer blends, polymer composites, and filled polymers: synthesis, properties, application. New York: Nova Science Publishers.

8. Galante, M. J., Mandelkern, L., \& Alamo, R. G. (1998). The crystallization of blends of different types of polyethylene: The role of crystallization conditions. Polymer, 39(21), 51055119. http://dx.doi.org/10.1016/S0032-3861(97)10162-8.

9. American Society for Testing and Materials. (2008). ASTM D6988 - 08: standard guide for determination of thickness of plastic film test specimens. West Conshohocken: ASTM.

10. Associação Brasileira de Normas Técnicas. (2008). ABNT NBR 15560 - 1: filmes plásticos agrícolas para cultivo protegidoParte 1: cobertura de estufas. São Paulo: ABNT.

11. Han Jiang et al (1999). Influence of surface roughness and contact load on friction coefficient and scratch behavior of 
thermoplastics olefins. Advances in Polymer Technology, 18(2), 171-180.

12. American Society for Testing and Materials. (1995). ASTM D1894-11e1: standard test method for static and kinetic coefficients of friction of plastic film and sheeting. West Conshohocken: ASTM.

13. American Society for Testing and Materials. (1999). ASTM D3418 - 99: standard test method for transition temperatures of polymers by differential scanning calorimetry. West Conshohocken: ASTM.

14. American Society for Testing and Materials. (2009). ASTM E2015 - 04: standard guide for preparation of plastics and polymeric specimens for microstructural examination. West Conshohocken: ASTM.

15. Brydson, J. A. (1999). Plastics materials (7th ed.). Wobum: Butterworth-Heinemann.

16. American Society for Testing and Materials. (2003). ASTM D1746 - 03: standard test method for transparency of plastic sheeting. West Conshohocken: ASTM.

17. American Society for Testing and Materials. (2000). ASTM D1003 - 00: standard test method for haze and luminous transmittance of transparent plastics. West Conshohocken: ASTM.

18. American Society for Testing and Materials. (1997). ASTM D2457 - 97: standard test method for specular gloss of plastic films and solid plastics. West Conshohocken: ASTM.

19. Progelhof, R. C., \& Throne, J. L. (1993). Polymer engineering principles: properties, process, test for design. New York: Hanser.

20. Blass, A. (1988). Processamento de polímeros. Florianópolis: Editora da Universidade Federal de Santa Catarina.

21. Martínez-Salazar, J., Cuesta, M. S., \& Plans, J. (1991). On phase separation in high- and low-density polyethylene blends: 1. Melting-point depression analysis. Polymer, 32(16), 29842988. http://dx.doi.org/10.1016/0032-3861(91)90197-Q.

22. Ferreira, R. L. R. (2003). Assegurando qualidade na produção de geomembranas de PEAD. Revista Engenharia, 559, 137.

23. Ferreira, R. L. R., Rempel, F. M., \& Salles, C. A. (2013). Ferramentas de controle da qualidade são aplicadas na melhoria da distribuição da espessura de filmes tubulares de PEBD. Revista Plástico Industrial, 30-59.

24. Ferreira, R. L. R., Girotto, N., Salles, C. A., \& Santana, R. M. C. (2011). Estudo da influência da razão de sopro na estrutura e propriedades de filmes tubulares de PEBD. In Anais do IX Simpósio Argentino de Polímeros - SAP 2011 (pp. 18). Bahía Blanca: SAP.

25. Ferreira, R. L. R., Santos, B., Konarzewski, V. H. C., \& Santana, R. M. C. (2011). Avaliação da correlação de estrutura e propriedades de filmes biorientados de PEBD.
In Anais do VI Congreso Internacional de Materiales - CIM 2011 (pp. 1). Bogotá: CIM.

26. Willmouth, F. M. (1986). Transparency, translucency and gloss. In F. M. Willmouth (Ed.), Optical properties of polymers (pp. 265-333). London: Elsevier.

27. White, J. L., \& Cakmak, M. (1988). Orientation, crystallization, and haze development in tubular film extrusión. Advances in Polymer Technology, 8(1), 27-61. http://dx.doi.org/10.1002/ adv.1988.060080105.

28. Pucci, M. S., \& Shroff, R. N. (1986). Correlation of blown film optical properties with resin properties. Polymer Engineering and Science, 26(8), 569-575. http://dx.doi.org/10.1002/ pen.760260808.

29. Patel, R. M., Butler, T. I., Walton, K. L., \& Knight, G. W. (1994). Investigation of processing structure-properties relationships in polyethylene blown films. Polymer Engineering and Science, 34(19), 1506-1514. http://dx.doi. org/10.1002/pen.760341911.

30. Chanda, M., \& Roy, S. K. (1987). Plastic technology handbook. New York: Marcel Dekker Inc.

31. Michaeli, W. (1992). Extrusion dies for plastics and rubber (2nd ed.). Munich: Hanser Publishers.

32. Rauwendaal, C. (2001). Polymer extrusion (4th ed.). Munich: Carl Hanser Verlag.

33. Roman, A. (1995). Transformação do polietileno - PEBD. Editora Érica Ltda.

34. Harper, C. A. (2006). Handbook of plastic processes. Wiley - Interscience. http://dx.doi.org/10.1002/0471786586.

35. Tadmor, Z., \& Gogos, C. G. (2006). Principles of polymer processing (2nd ed.). Wiley - Interscience.

36. Cantor, K. (2006). Blown film extrusion: an introduction. Munich: Carl Hanser Verlag.

37. Rauwendaal, C. (1998). Polymer mixing, a self-study guide. Munich: Carl Hanser Verlag.

38. Rauwendaal, C. (1998). Understanding extrusion. Munich: Carl Hanser Verlag.

39. Rauwendaal, C. (2000). Statistical process control in injection molding and extrusion. Munich: Carl Hanser Verlag.

40. Rauwendaal, C., \& Noriega, P. (2001). Troubleshooting the extrusion process. Munich: Carl Hanser Verlag.

41. Rauwendaal, C., \& Cantor, K. (2001). Extrusion handbook. Munich: Carl Hanser Verlag.

42. Giles, H. F., Wagner, J. R., \& Mount, E. M. (2005). Extrusion: the definitive processing guide and handbook. Norwich: William Andrew, Inc.

Enviado: Nov. 11, 2013

Reenviado: Jul. 11, 2014

Aceito: Ago. 04, 2014 